\section{Luminance control of a small computer CRT display: A very cheap technique*}

\author{
ROBERT SEKULER and ROBERT ARMSTRONG \\ Northwestern University, Evanston, Illinois 60201
}

An inexpensive way to produce continuous variation in the luminance of a computer-controlled CRT display is described. One application of that system to the study of motion-analyzers in human vision is discussed briefly.

There is an increasing acceptance and use of minicomputer-controlled cathode-ray tube displays in research in vision and visual perception (Uttal, 1969; Haber, 1968; Mayzner, 1968). Often an investigator is interested in measuring the threshold value of some stimulus presented on the CRT screen. For a variety of reasons, a luminance threshold is a more desirable measure than are other measures, e.g., a duration threshold (Graham, 1958). An obvious way to vary the effective luminance of the CRT display is to vary the rate at which points on that screen are refreshed or to vary the number of times a given point is refreshed. Unfortunately, these schemes have serious deficiencies because they involve a confounding of a temporal variable with changes in luminance. Until now it has been difficult to place the luminance of the CRT directly under computer control. In this paper we report a good, fast, and above all, inexpensive way to accomplish this.

We will describe the technique as it has been developed for the most common variety of minicomputer of the Digital Equipment Corporation PDP-8 family. Slight modifications of our basic strategy will make it easily usable by people with other kinds of computers. Although our technique is not logically specific to the configuration of our lab's computer, it is convenient to describe the particular way we have implemented it. Most of our interfacing to the PDP-8 computer is done using Digital Equipment Corporation's general-purpose interface package, the AX-08, or laboratory peripheral. In addition to providing digital and analog inputs to the computer, the AX-08 controls the position and intensity of the CRT beam via three digital to-analog converters. Consider the general operation of the CRT intensifying circuit on the AX-08. When a point is to be plotted on the CRT, the beam is positioned in $\mathrm{X}$ and $\mathrm{Y}$ coordinates by

* The development of the system described herein was supported by a grant from the Eye Institute of the National Institutes of Health, EY-00321. voltages loaded from appropriate buffers. The beam intensity at that point is determined by a one-shot ( $R C$ network) which feeds into a voltage integrator. The longer the time constant of the one-shot, the higher the voltage the integrator will put into the CRT beam. This, of course, will result in a spot of higher luminance on the CRT screen. The AX-08 has, as part of its XR option, three separate one-shots, any one of which, under program control, can be tied into the scope intensity control circuit. These one-shots are nominally adjusted for pulse widths of 500 nsec (dim display level), 2.5 microsec (a medium display level), and 10 microsec (the brightest display level). Our technique for producing continuous variation in CRT luminance has been to place a precision potentiometer in parallel with the RC network of that one-shot which is normally used for the dimmest display level. ${ }^{1}$ This potentiometer is mounted on the shaft of a bidirectional stepping motor capable of operating at 75 steps/sec. The digital logic which controls the stepping motor is activated by pulses derived from the easily interfaced outputs of the AX-08 itself. Although this system is extremely reliable and fast, we have taken the precaution of having the computer monitor the actual changes in the stepping motor's position via Schmitt trigger inputs to the AX-08. An alternative and somewhat more elegant way to achieve much the same thing would be to have the external potentiometer used in the one-shot network mechanicaliy ganged to another precision potentiometer. Voltages across that second pot could be input to one of the analog lines of the AX-08 and the absolute position of the pot at any time instantly determined by sampling the voltage with the analog-to-digital converters of the AX-08. This implementation would also make it possible to substitute an ordinary nonstepping reversible motor with a good brake for the stepping motor we chose.

We are now using a 10-turn precision pot of 5,000-ohm resistance. Since the stepping motor has 200 steps/revolution, we have virtually continuous (though actually incremental) control of display luminance whenever a program calls for the dimmest display level. The CRT luminance is linear with the resistance of the precision pot over a range of approximately 2 log units variation in luminance. In our particular system, with its setting of the internal resistance of the one-shot, the scope used (Tektronix RM503) and the phosphor of the CRT (P-31), the relationship between luminance and resistance becomes nonlinear at approximately $200 \mathrm{ohms}$. By choosing appropriate filters for the CRT and maintaining the correct ambient illumination in the experimental room, we have been able to ensure that the S's threshold for the displays we present is kept within the linear portion of our range. Adjustment of the CRT phosphor, the internal resistance of the one-shot, or the scope beam current regulation could probably extend the useful range of our system to considerably more than its present 2 log units. The total cost of the luminance control system is under $\$ 100$ for the stepping motor, digital controls, and precision pot. Since most laboratories have supplies of bidirectional stepping motors that can be cannibalized from operant conditioning apparatus or other sources, the more likely cost of the system is that of the potentiometer alone, about $\$ 10$. Six additional computer instructions are required to produce the logarithmically arrayed luminance changes that most applications require.

The flexibility and ease of the system can best be understood from a brief description of the first experiments in which it has been used. We have been interested in the response of visual mechanisms sensitive to various parameters of motion. In one experiment, the $\mathrm{S}$ sat in front of the CRT, fixed on a biting board, and observed for several seconds a superthreshold pattern of vertical bars which move across the CRT at a velocity of $5 \mathrm{deg} / \mathrm{sec}$. This pattern is about $2.4 \log$ units above threshold and is displayed by means of an instruction to the computer to use the one-shot for normal brightness levels. Since we are bypassing the "dim"-level one-shot, this adaptation pattern will be presented at a constant brightness level which is independent of the position of the precision pot. This adaptation display is turned off and instantly replaced by a dimmer, test display. The computer is programmed to present a pattern of vertical bars either in the top half of the field (above a fixation point) or in the bottom half of the field. The luminance of these test bars is 
controlled by the external precision pot and the one-shot normally used for the dim display level. All points in this test pattern are plotted at the same luminance level. The S's task is to identify in which half of the field the test pattern was presented, a two-alternative spatial forced choice. The test field is presented for only $250 \mathrm{msec}$, and then the normal, fixed-level adaptation pattern returns. The S's response is input to the computer from a teletypewriter. If that response is wrong, the computer causes the stepping motor to turn the pot through enough steps so that the next time the test is given it will have a luminance $0.03 \mathrm{log}$ units higher. When the $S$ gets three responses in a row correct, the luminance is decreased by that same amount for the next presentation. This procedure, a combination of forced choice and tracking, estimates the 0.79 point on the psychometric function (Wetherill \& Levitt, 1965). Thresholds are measured quickly and seem just as stable and repeatable day to day as those measured using a comparable procedure in a standard non-CRT Maxwellian view system (Pantle \& Sekuler, 1969).

We have replicated earlier results with the finding that when the adaptation field moves left to right, a test pattern moving in that same direction has a threshold about 0.40 $\log$ units higher than one which moves in the opposite direction (Pantle \& Sekuler, 1969). This threshold difference between same and opposite conditions is called "direction-specific adaptation" since it is assumed to reflect the extent to which a moving pattern adapts visual mechanisms specialized for response to its direction of motion. Several novel findings have emerged from the computer display experiments that could not have been performed in other ways. Among the most intriguing is the following. Before plotting any given point in our display of vertical lines, the computer samples the voltage present at one of the analog inputs. This voltage is supplied from a standard random-noise generator and varies over time in an approximately Gaussian fashion. The computer adds that random voltage level to the buffer, in which is stored the $\mathrm{X}$ coordinate of the point which it is to plot next. By varying the mean voltage level present at the analog input, we can cause the computer to display any pattern from uniform vertical lines (with zero voltage present) to displays of points that are random in the $\mathrm{X}$ dimension (with about $0.2 \mathrm{~V}$ present). Of course, we can also produce displays with intermediate amounts of randomness, and the same technique can also be applied to $\mathrm{Y}$ coordinates as well. If one substitutes such a random pattern for the usual adaptation pattern of vertical lines, that random pattern has far less differential effect on the luminance threshold for test lines that move in the same direction vs those that move in the opposite direction. $\mathrm{Tr}^{\circ}$, reduction in direction-specific adaptation argues that there is a functional segregation of human visual motion analyzers according to the configurational properties of their optimal stimuli. This is a prospect that we are currently exploring with our computer-controlled CRT display.

\section{REFERENCES}

GRAHAM, C. H. Sensation and perception in an objective psychology. Psychological Review, 1958, 65, 65-76.

HABER, R. N. An on-line computer in a visual perception laboratory. Behavior Research Methods \& Instrumentation. $1968,1,86-93$.

MAYZNER, M. S. The research potential of a computer-based cathode-ray tube display system. Behavior Research Methods \& Instrumentation, 1968, 1, 41-43.

PANTLE, A. \& SEKULER, R, Contrast response of human visual mechanisms sensitive to orientation and direction of motion. Vision Research, 1969, 9. 397-406.

UTTAL, W. R. Buggywhips, whalebones. and clipboards. American Psychologist. $1969,24,202-206$.

WETHERILL, G. B., \& LEVITT, H. Sequential estimation of points on a psychometric function. British Journal of Mathematical \& Statistical Psychology, $1965,18,1-10$.

\section{NOTE}

1. this arrangement, suggested to us by Roy Van Liew, also protects the one-shot circuit. 